Pacific Journal of Mathematics

jITNEY STABILITY OF SOLVABILITY 


\title{
WHITNEY STABILITY OF SOLVABILITY
}

\author{
John K. Beem And Phillip E. Parker
}

\begin{abstract}
The set of symbols which are both of real principal type and pseudoconvex is shown to be open in the Whitney topology on the space of symbols of order $k$. This yields sufficient conditions for the stability of solvability of pseudodifferential equations.
\end{abstract}

1. Introduction. In a previous paper [3] we studied the stability of solvability for pseudodifferential equations of real principal type in the $F \mathscr{D}$ topology of Michor [9] and in an analogous topology we defined, the $F \mathscr{S}$ topology. In particular, we considered the stability of real principal type and of pseudoconvexity in the space of principal symbols of order $k$. These two conditions together imply solvability and hence their stability implies stability of solvability where the conditions hold.

We say that a condition is stable for a given topology if the set where the condition holds is an open set in that topology. A condition is stable at a point if it is satisfied on an open neighborhood of that point. Of course, stability in one topology implies stability in all finer topologies. In the present paper we consider the stability of real principal type and of pseudoconvexity in the $C^{r}$-coarse and $C^{r}$-fine topologies on the space of principal symbols of order $k$.

In $\$ 2$ we first review the $C^{r}$-coarse and $C^{r}$-fine topologies. We then give examples showing that neither real principal type nor pseudoconvexity is stable in the $C^{r}$-coarse, $r \geq 0$, or $C^{0}$-fine topologies, and that real principal type is not stable in any $C^{r}$-fine topology.

In $\$ 3$ we first outline and then give the complete proof of Whitney or $C^{r}$-fine, $r \geq 1$, stability of real principal type and pseudoconvexity jointly. This establishes the $C^{r}$-fine stability of solvability of a pseudodifferential equation with a corresponding principal symbol which is both of real prinicpal type and pseudoconvex.

In $\$ 4$ we consider pseudoriemannian manifolds $(X, \beta)$ of $\operatorname{dim} \geq 3$. If $\beta$ is given contravariantly, then $\beta$ is naturally a principal symbol of order 2 . We begin $\S 4$ with some new results on sectional curvature. In general, everywhere negative timelike sectional curvature is not a $C^{r}$-fine stable condition for any $r \geq 0$. However, if the Riemann-Christoffel curvature $R$ satisfies a nonvanishing requirement on all timelike and null planes, then 
we show everywhere negative timelike sectional curvature is $C^{2}$-fine stable. Using this result and some results from [3] we finish $\$ 4$ with some applications of Whitney stability.

Most of our notations and conventions are standard, and are the same as in [3]. Except in part of $\S 4$, we regard a pseudoriemannian structure tensor $\beta$ as given in contravariant form (i.e., as a $(2,0)$ tensor). $\mathscr{E}$ denotes the real valued smooth functions, $\mathscr{D}$ those with compact support, and $\mathrm{Smbl}_{r}$ the principal symbols of order $r$. Manifolds are smooth, paracompact, connected, and usually noncompact.

2. Topologies and instability. In this section we discuss the $C^{r}$ coarse and $C^{r}$-fine topologies for $r \geq 0$. References to $F \mathscr{D}$ and $F \mathscr{S}$ topologies will be for comparison only, and we refer the reader to [3] for details. We shall give examples to show that real principal type is neither $C^{r}$-coarse nor $C^{r}$-fine stable for $r \geq 0$, and that pseudoconvexity is neither $C^{r}$-coarse, $r \geq 0$, nor $C^{0}$-fine stable.

First we consider the $C^{r}$-coarse or $S$ chwartz $C^{r}$ topology. Intuitively, this is the topology of uniform convergence of the function and its derivatives up through order $r$ on compact sets. Let $\left\{K_{i}\right\}$ be a countable family of compact sets in $X$ such that $K_{i} \subseteq \stackrel{\circ}{K}_{i+1}$ and $X=\bigcup K_{i}$. Choose any compatible fiber metric $d_{r}$ on $J^{r}(X)$ and define seminorms

$$
\mathscr{P}_{i}(\phi):=\sup _{k \leq r} \sup _{K_{i}} d_{k}\left(j_{x}^{k}(\phi), 0_{x}\right) \text {. }
$$

This defines a vector topology on $\mathscr{E}$ which makes it a nuclear Fréchet space. The usual Schwartz or $C^{\infty}$-coarse topology is the union of all these $C^{r}$-coarse topologies.

The other main topology is the $C^{r}$-fine or Whitney $C^{r}$ topology. Whereas the Schwartz $C^{r}$ topology gives no control of the convergence at $\infty$, the Whitney $C^{r}$ topology gives arbitrary amounts of such control. In doing so, however, it is no longer a vector topology on $\mathscr{E}$; thus it cannot be described in terms of seminorms. To compare these topologies, recall that $j^{r}: \mathscr{E} \rightarrow C\left(X, J^{r}(X)\right)$. Now on the latter space we can place either the compact-open or the graph topology. Via $j^{r}$, these induce the Schwartz, respectively Whitney, $C^{r}$ topology on $\mathscr{E}$; see [9] or [11] for details. In terms of a fiber metric $d_{r}$ on $J^{r}(X)$, we can describe the Whitney $C^{r}$ topology as follows. Let $\varepsilon \in C(X,(0, \infty))$; then

$$
\mathcal{N}(\phi, r, \varepsilon):=\left\{\psi \in \mathscr{E} ; d_{r}\left(j^{r} \phi, j^{r} \psi\right)<\varepsilon\right\}
$$

is a Whitney $C^{r}$ neighborhood of $\phi$, and as $\varepsilon$ varies we obtain a basis of Whitney $C^{r}$ neighborhoods of $\phi$. Later we shall also need a local version 
of these neighborhoods. In addition to the above data, let $V \subseteq X$ be open and define

$$
\mathscr{N}(\phi, r, \varepsilon, V):=\left\{\psi \in \mathscr{E} ; d_{r}\left(j^{r} \phi, j^{r} \psi\right)<\varepsilon \text { on } V\right\} .
$$

When $V=X$ we shall omit it from the notation to coincide with the first definition. For convenience, we allow the following abuse of notation: if $X$ is a bundle over $Y$, then $V$ may be a subset of $Y$ and "on $V$ " is replaced by "over $V$ ".

If we define $F \mathscr{D}^{r}$ and $F \mathscr{S}^{r}$ topologies by analogy, then we can list all four in order from coarsest to finest:

$$
\text { Schwartz } C^{r}, F \mathscr{S}^{r} \text {, Whitney } C^{r}, F \mathscr{D}^{r} \text {. }
$$

Thus $C^{r}$-coarse stability $\Rightarrow F \mathscr{S}^{r}$ stability $\Rightarrow C^{r}$-fine stability $\Rightarrow F \mathscr{D}^{r}$ stability.

Since principal symbols are positive homogeneous in the fiber variables, the obvious $C^{r}$ topologies on them are discrete. Thus we shall modify them as in [3] to take homogeneity into account. Letting $h$ be an auxiliary complete Riemannian (positive definite) metric on $X$, a symbol is completely determined by its order of homogeneity $k$ and its restriction to the $h$-unit cosphere bundle $S^{*} X$. Thus, given $k$, there is a bijection $I$ : $\mathscr{E}\left(S^{*} X\right) \rightarrow \operatorname{Smbl}_{k}(X)$ between the smooth functions on $S^{*} X$ and the space of principal symbols of order $k$. We shall say that a set $U \subseteq$ $\operatorname{Smbl}_{k}(X)$ is open in a $C^{r}$ topology iff the corresponding set $I^{-1}(U) \subseteq$ $\mathscr{E}\left(S^{*} X\right)$ is open in that $C^{r}$ topology on $\mathscr{E}\left(S^{*} X\right)$.

We now proceed with the examples mentioned above and to which we referred in [3].

EXAMPLE 2.1. Real principal type is not $C^{r}$-fine stable, $r \geq 0$. Here we use the example following Proposition 3.2 in [3]. For the convenience of readers, we repeat the essential parts here. Let $X_{0}$ be the open Möbius strip $\{(x, y) ;-\infty<x<\infty, 0 \leq y \leq 4\}$ with the identification $(x, 0) \sim$ $(-x, 4)$ and the metric tensor $g_{0}=\xi_{1} \xi_{2}$ (i.e., $d s^{2}=d x d y$ ). The bicharacteristic curves of $g_{0}$ lie on the null geodesics of $g_{0}$ which are the Euclidean lines $y=$ const. and the circles $|x|=$ const. The manifold $X$ will be $X_{0}$ less the two closed half lines $L_{1}=\{(x, 1) ; x \leq 0\}$ and $L_{2}=\{(x, 3)$; $x \geq 0\}$. The metric tensor on $X$ will be $g=g_{0} \mid X$. The Lorentzian manifold $(X, g)$ has no imprisoned null geodesics and hence $g$ is a symbol of real principal type. On the other hand, any $C^{r}$-fine neighborhood $U(g)$ of $g$ in $\operatorname{Smbl}_{2}(X)$ will contain some $g^{\prime}$ which has a periodic null geodesic.

EXAMPLE 2.2. Real principal type and pseudoconvexity are neither jointly nor separately $C^{0}$-fine stable. Let $X=\mathbf{R}^{4}$ with the pseudoeuclidean 
structure $\beta:=\xi_{1}^{2}+\xi_{2}^{2}-\xi_{3}^{2}-\xi_{4}^{2}$. Notice that the plane $\Pi=\left\{x^{1}=x^{3}\right.$, $\left.x^{2}=x^{4}\right\}$ is totally null; hence any curve of the form $\left(\gamma_{1}, \gamma_{2}, \gamma_{1}, \gamma_{2}\right)$ is null. The Hamiltonian vector field of $\beta$ is

$$
H^{\beta}=\sum_{i=1}^{2} 2 \xi_{i} \frac{\partial}{\partial x^{i}}-\sum_{i=3}^{4} 2 \xi_{i} \frac{\partial}{\partial x^{i}},
$$

which has solution curves

$$
\begin{aligned}
& \gamma_{1}(t)=\left(2 c_{1} t+a_{1}, c_{1}\right) \\
& \gamma_{2}(t)=\left(2 c_{2} t+a_{2}, c_{2}\right) \\
& \gamma_{3}(t)=\left(2 c_{3} t+a_{3}, c_{3}\right) \\
& \gamma_{4}(t)=\left(2 c_{4} t+a_{4}, c_{4}\right)
\end{aligned}
$$

in induced cotangent coordinates $(x, \xi)$. If we choose $c_{1}=-c_{3}, c_{2}=c_{4}$, $a_{1}=a_{3}$, and $a_{2}=a_{4}$, then the curve

$$
\gamma(t)=\left(2 c_{1} t+a_{1}, 2 c_{2} t+a_{2}, 2 c_{1} t+a_{1}, 2 c_{2} t+a_{2}, c_{1}, c_{2},-c_{1},-c_{2}\right)
$$

is a bicharacteristic strip.

Let $\varepsilon: \mathbf{R}^{4} \rightarrow(0, \infty)$ define a $C^{0}$-fine neighborhood $\mathscr{N}(\beta, 0, \varepsilon)$ of $\beta$ and let $\varepsilon^{\prime}: \mathbf{R}^{2} \rightarrow(0, \infty)$ be a continuous function such that

$$
\begin{aligned}
0 & <\varepsilon^{\prime}\left(x^{1}, x^{2}\right) \\
& <\inf \left\{\varepsilon\left(x^{1}, x^{2}, x^{3}, x^{4}\right) ;\left|\left(x^{1}, x^{2}, x^{3}, x^{4}\right)-\left(x^{1}, x^{2}, x^{1}, x^{2}\right)\right|<1\right\},
\end{aligned}
$$

where $|\cdot|$ denotes the usual Euclidean norm in $\mathbf{R}^{4}$. Intuitively, $\varepsilon^{\prime}$ is smaller at points in $\mathbf{R}^{2}$ than $\varepsilon$ is at all points within distance 1 of the corresponding points of $\Pi \subseteq \mathbf{R}^{4}$. Now choose any Riemannian (positive definite) metric $g \in \mathscr{N}\left(g_{0}, 0, \varepsilon^{\prime}\right)$, where $g_{0}$ is the usual Euclidean metric on $\mathbf{R}^{2}$. Regard $T^{*} \Pi=\mathbf{R}^{2} \oplus \mathbf{R}^{2}$ and define $\beta^{\prime}=g \oplus(-g)$ on $T^{*} \Pi$. Then on $\Pi$,

$$
\beta^{\prime} \in \mathscr{N}(\beta|\Pi, 0, \varepsilon| \Pi) \text {. }
$$

Finally extend $\beta^{\prime}$ from $\Pi$ to $\mathbf{R}^{4}$ with $\beta^{\prime} \in \mathscr{N}(\beta, 0, \varepsilon)$.

Example 5.1 of [2] can now be modified to show that $g$ can be chosen so that $\beta^{\prime}$ is not of real principal type and so that $\mathbf{R}^{4}$ is not $\beta^{\prime}$-pseudoconvex. We change $g_{0}$ on small discs centered at $(1,0),(0,1)$, and $(0,-1)$ so that a closed geodesic joining these three points is introduced, and then change $g_{0}$ on small discs $D_{k}$ centered at $(k, 0)$ for $k=2,3,4, \ldots$, so that geodesics from $(0,-1)$ to $(0,1)$ are introduced which pass through each $D_{k}$. To complete our description of $g$, we declare that outside these discs the geodesics are the usual straight lines. It follows that we can produce a desired $\beta^{\prime}$ which is $C^{0}$-fine close to $\beta$, but not $C^{1}$-fine close. 
EXAMPLE 2.3. Real principal type is not $C^{r}$-coarse stable, $r \geq 0$. Let $X=\mathbf{R}^{3}$ and $\eta$ the usual Minkowski structure. For any compact $K \subseteq \mathbf{R}^{3}$, let $B_{K}$ be a closed Euclidean tube centered on the $t$-axis which contains $K$ in its interior $\stackrel{\circ}{K}_{K}$. Choose $\psi \in \mathscr{E}\left(\mathbf{R}^{3}\right)$ such that:

1. $\psi \equiv 0$ outside $B_{K}$;

2. $\psi \equiv 1$ on a closed tube which contains $K$;

$3.0 \leq \psi \leq 1$

4. $\psi$ is a function of $r$ only, and is nonincreasing.

We are using cyclindrical coordinates $(t, r, \theta)$ where $r$ is the Euclidean distance from the $t$-axis. For our symbol $\beta$, we choose the line element

$$
d s^{2}=\psi\left(d t^{2}-d r^{2}-r^{2} d \theta^{2}\right)+(1-\psi)\left(d t d \theta-d r^{2}-r^{2} d \theta^{2}\right) .
$$

By adjusting the size of $B_{K}$ relative to $\{\psi \equiv 1\}$ and the derivatives of $\psi$ in between, we can produce such a $\beta$ in any $C^{r}$-coarse neighborhood of $\eta$. $\beta$ is not of real principal type, since it has closed null geodesics outside $B_{K}$.

EXAmple 2.4. Pseudoconvexity is not $C^{r}$-coarse stable, $r \geq 0$. This is obtained by using a cut-off function as in Example 2.3 to modify Example 2.2; we omit the straightforward details.

3. Whitney stability. In this section we consider the stability of real principal type and of pseudoconvexity in the $C^{1}$-fine (Whitney) topology. Let $p$ be a principal symbol of order $k \geq 0$ which is of real principal type and psuedoconvex. We show that all principal symbols of the same order $k$ which are sufficiently close to $p$ in the $C^{1}$-fine topology are also of real principal type and pseudoconvex. Thus the set of principal symbols which are both pseudoconvex and of real principal type is open in the space of principal symbols using the $C^{1}$-fine topology.

We always assume the manifold $X$ is not compact because no symbol is of real principal type of a compact manifold. Also, pseudoconvexity is trivially true for symbols on compact manifolds.

Examples 2.1 and 2.2 show that the conditions of real principal type and pseudoconvexity fail to be $C^{0}$-fine stable. The fact that real principal type fails to be stable in the $C^{r}$-fine topology for all $0 \leq r \leq \infty$ (cf. Example 2.1) is somewhat surprising since the bicharacterics come from the Hamiltonian vector field $H^{p}$ which only involves the first derivatives of $p$. One would expect $a$ priori that real principal type would be $C^{1}$-fine stable.

The basic tool used in establishing the $C^{1}$-fine stability of the two conditions jointly is a standard estimate from differential equations $[4, \mathrm{p}$. 155]. This result implies that when $p$ and $p^{\prime}$ are principal symbols of the 
same order $k$ which have values and first derivatives which are close on the unit cosphere bundle $S^{*} K$ of some compact $K \subset X$, then bicharacteristic curves of $H^{p}$ and $H^{p^{\prime}}$ will remain close in $K$ for some compact domain $[0, a]$ provided that the initial values of the corresponding bicharacteristic strips are chosen close. The idea is to use pseudoconvexity to construct an expanding sequence $\left\{A_{n}\right\}$ of compact sets: at each step, take the pseudoconvex hull of the preceding step; this has compact closure by pseudoconvexity; finally, enlarge it if necessary to enclose a suitable neighborhood of the previous step. Then use the differential equations estimate above and real principal type to choose a corresponding sequence $\left\{\varepsilon_{n}\right\}$ of positive numbers: intuitively, $\varepsilon_{n}$ measures how far $p^{\prime}$ can be from $p$ on $A_{n}$ and still be of real principal type and pseudoconvex. The pairs $\left\{A_{n}, \varepsilon_{n}\right\}$ determine $C^{1}$-fine neighborhoods of $p$, any one of which will serve. The actual construction is more complicated because we must keep careful track of the bounds in order that they interweave properly. In Lemma 3.1 we show how to achieve all but one necessary bound in a uniform manner in the index $n$. We then choose a neighborhood, and obtain the other necessary bound in Lemma 3.2. Finally, Theorem 3.3 assembles the parts.

We now begin the technical details. Recall that the space of principal symbols of order $k$ is denoted by $\operatorname{Smbl}_{k}(X)$. If $\gamma_{1}$ and $\gamma_{2}$ are two complete bicharacteristic strips of $p \in \operatorname{Smbl}_{k}(X)$ with $\gamma_{1}(0)=(x, \xi)$ and $\gamma_{2}(0)=$ $(x, \lambda \xi)$ for some positive constant $\lambda$ (i.e., $\gamma_{1}$ and $\gamma_{2}$ start over the same $x \in X$ and in the same codirection), then the bicharacteristic curves $\pi \circ \gamma_{1}$ and $\pi \circ \gamma_{2}$ only differ by a reparametrization. Thus for our purposes it is sufficient to consider only one bicharacteristic curve for each codirection at each point $x \in X$.

As in $\S 2$, let $h$ be an auxiliary complete Riemannian metric on $X$ and use it to topologize $\operatorname{Smbl}_{k}(X)$ with the $C^{1}$-fine topology from $\mathscr{E}\left(S^{*} X\right)$. The metric tensor $h$ induces a complete metric distance function $d_{h}$ on $X$. The Sasaki lift of $h$ to $T^{*} X$ induces a distance function on $T^{*} X$ and the restriction of this distance function to the $h$-unit cosphere bundle will be denoted by $d_{0}$. There is also an induced distance function on $J^{r}\left(S^{*} X\right)$ which will be denoted by $d_{r}$. As in $\S 2$, we use $\mathscr{N}(\phi, r, \varepsilon, V)$ to denote a basic $C^{r}$-fine neighborhood of $\phi$ over the set $V$. Hence,

$$
\begin{array}{r}
\mathscr{N}(\phi, r, \varepsilon, V)=\left\{\psi \in \mathscr{E}\left(S^{*} X\right) ; d_{r}\left(j^{r} \phi(x, \xi), j^{r} \psi(x, \xi)\right)\right. \\
\text { for all } \left.(x, \xi) \in S^{*} V\right\}
\end{array}
$$

where $\varepsilon: S^{*} V \rightarrow \mathbf{R}$ is a continuous positive valued function, $\phi \in \mathscr{E}\left(S^{*} X\right)$, and $V \subseteq X$. 
The distance function $d_{0}$ may be used to recover the topology on the cosphere bundle $S^{*} X$. The open balls of $S^{*} X$ are given by $B\left(v_{0}, \delta\right)=$ $\left\{v \in S^{*} X ; d_{0}\left(v, v_{0}\right)<\delta\right\}$ where $v_{0} \in S^{*} X$ and $\delta>0$ are arbitrary.

The bicharacteristic equations for the principal symbol $p$ involve the first derivatives of $p$ with respect to $x_{i}$ and $\xi_{i}$, but no higher order derivatives. Thus, if $\gamma:[0, a] \rightarrow T^{*} x$ is a fixed bicharacteristic strip of $p$ in $T^{*} X$ with $\gamma(0)=v_{0} \in S^{*} X$ and if $\tilde{\gamma}:[0, a] \rightarrow T^{*} X$ is a bicharacteristic strip of $p^{\prime}$ with $\tilde{\gamma}(0)=v$, then $d_{h}(\pi \circ \gamma(t), \pi \circ \tilde{\gamma}(t))<1$ for all $0 \leq t \leq a$ provided that $v$ is chosen sufficiently close to $v_{0}$ and that $p^{\prime}$ is sufficiently close to $p$ in the $C^{1}$-fine topology. Using this fact and the compactness of $S^{*} K_{1}$ when $K_{1}$ is compact, we obtain the following lemma.

LEMMA 3.1. Assume $K_{1}$ is a compact set contained in the interior of the compact set $K_{2}$. Let $V$ be an open set containing $K_{2}$ and let $p$ be a symbol in $\operatorname{Smbl}_{k}(X)$ which is of real principal type. There exist cotangent vectors $v_{1}, \ldots, v_{m} \in S^{*} K_{1}$ and positive constants $\delta_{1}, \ldots, \delta_{m}, a_{1}, \ldots, a_{m}, \varepsilon$ such that if $p^{\prime} \in \mathscr{N}(p, 1, \varepsilon, V)$ then the following hold:

1. if $\gamma$ is a complete bicharacteristic strip of $p$ with $\gamma(0) \in B\left(v_{i}, \delta_{i}\right)$, then $\pi \circ \gamma\left(\left[0, a_{i}\right]\right) \subset V$ and $\pi \circ \gamma\left(a_{i}\right) \in V \backslash K_{2}$;

2. if $\tilde{\gamma}$ is a complete bicharacteristic strip of $p^{\prime}$ with $\tilde{\gamma}(0) \in B\left(v_{i}, \delta_{i}\right)$, then $\pi \circ \tilde{\gamma}\left(\left[0, a_{i}\right]\right) \subset V$ and $\pi \circ \tilde{\gamma}\left(a_{i}\right) \in V \backslash K_{2}$;

3. two complete bicharacteristic strips $\gamma$ and $\tilde{\gamma}$ of $p$ and $p^{\prime}$, respectively, with $\gamma(0), \tilde{\gamma}(0) \in B\left(v_{i}, \delta_{i}\right)$ satisfy $d_{h}(\pi \circ \gamma(t), \pi \circ \tilde{\gamma}(t))<1$ for all $0 \leq t \leq$ $a_{i}$;

4. $\bigcup_{i} B\left(v_{i}, \delta_{i}\right) \supseteq S^{*} K_{1}$.

If $x_{0} \in X$ and $A$ is some subset of $X$, then the $d_{h}$ distance from $x_{0}$ to $A$ is given by $d_{h}\left(x_{0}, A\right)=\inf \left\{d_{h}\left(x_{0}, y\right) ; y \in A\right\}$. Using $d_{h}$ we now define an increasing sequence $A_{0}, A_{1}, A_{2}, \ldots$, of compact sets which exhausts the $p$-pseudoconvex space $X$. Fixing $x_{0} \in X$, let $A_{0}=\left\{x_{0}\right\}$ and $A_{1}=\{x \in X$; $\left.d_{h}\left(x_{0}, x\right) \leq 2\right\}$. If $A_{0}, A_{1}, \ldots, A_{n}$ have been defined, let $A_{n+1}$ be a compact set containing the pseudoconvex hull of $A_{n}$ with $d_{h}\left(x, X \backslash A_{n+1}\right)>2$ for all $x \in A_{n}$; i.e., if $\gamma:[a, b] \rightarrow M$ is a segment of a bicharacteristic strip of $p$ in $T^{*} X$ with both endpoints $\pi \circ \gamma(a), \pi \circ \gamma(b) \in A_{n}$, then $\pi \circ \gamma \|[a, b]$ lies in the interior of $A_{n+1}$. [The pseudoconvexity of $p$ implies that the sequence $\left\{A_{n}\right\}$ may be constructed.]

We now construct a sequence $\left\{\varepsilon_{n}\right\}$ of monotonic nonincreasing positive constants. Let $\varepsilon_{-3}>0$ and let $\varepsilon_{-2}$ be the minimum of $\varepsilon_{-3}$ and the $\varepsilon$ of Lemma 3.1, using $K_{1}=A_{1}, K_{2}=A_{5}$ and $V=$ interior $\left(A_{6}\right)$. Assume $p^{\prime} \in \mathscr{N}(p, 1, \varepsilon, V)$ and $v_{0} \in S^{*} K_{1}$. If $\gamma$ and $\tilde{\gamma}$ are bicharacteristic strips of 
$p$ and $p^{\prime}$, respectively, with $v_{0}=\gamma(0), \tilde{\gamma}(0) \in B\left(v_{i}, \delta_{i}\right)$ then by Lemma 3.1 both $\pi \circ \gamma\left(a_{i}\right)$ and $\pi \circ \tilde{\gamma}\left(a_{i}\right)$ lie in $A_{6} \backslash A_{5}$ for some $a_{i}$. Furthermore, $d_{h}(\pi \circ \gamma(t), \pi \circ \tilde{\gamma}(t))<1$ for all $0 \leq t \leq a_{i}$. Using this fact and the fact that any bicharacteristic strip $\gamma$ of $p$ with $\pi \circ \gamma\left(t_{1}\right), \pi \circ \gamma\left(t_{2}\right) \in A_{2}$ must satisfy $\pi \circ \gamma\left(\left[t_{1}, t_{2}\right]\right) \subset A_{3}$, we find that any bicharacteristic $\tilde{\gamma}$ of $p^{\prime}$ which satisfies $\pi \circ \tilde{\gamma}([0, b]) \subset A_{5}, \pi \circ \tilde{\gamma}(0) \in A_{1}$, and $\pi \circ \tilde{\gamma}(b) \in A_{1}$ must also satisfy $\pi \circ \tilde{\gamma}([0, b]) \subset A_{4}$.

Assume now that $\varepsilon_{-3}, \varepsilon_{-2}, \ldots, \varepsilon_{n-3}$ have been defined. Let $\varepsilon_{n-2}$ be the minimum of $\varepsilon_{n-3}$ and the $\varepsilon$ of Lemma 3.1 using $K_{1}=A_{n+1}, K_{2}=A_{n+5}$ and $V=\operatorname{interior}\left(A_{n+6}\right)$. Recursively, this defines the sequence $\left\{\varepsilon_{n}\right\}$. Let $\delta: X \rightarrow \mathbf{R}$ be a positive valued continuous function such that $\delta(x)<\varepsilon_{n}$ for each $x \in A_{n} \backslash A_{n-1}$.

LEMMA 3.2. Assume $p$ is of real principal type and pseudoconvex, and let $p^{\prime} \in \mathscr{N}(p, 1, \delta, X)$. If $\tilde{\gamma}:(a, b) \rightarrow T^{*} X$ is a complete bicharacteristic strip of $p^{\prime}$, there do not exist values $a<t_{1}<t_{2}<t_{3}<b$ with $\pi \circ \tilde{\gamma}\left(t_{1}\right) \in A_{n}$, $\pi \circ \tilde{\gamma}\left(t_{3}\right) \in A_{n}$ and $\pi \circ \tilde{\gamma}\left(t_{2}\right) \in A_{n+4} \backslash A_{n+3}$.

Proof. We may assume without loss of generality that $\pi \circ \tilde{\gamma} \|\left[t_{1}, t_{3}\right] \subset$ $A_{n+4} \backslash A_{n-1}$ and that $\tilde{\gamma}\left(t_{1}\right) \in S^{*} A_{n}$. Let $\gamma$ be a complete bicharacteristic strip of the original symbol $p$ with $\gamma\left(t_{1}\right), \tilde{\gamma}\left(t_{1}\right) \in B\left(v_{i}, \delta_{i}\right)$ as in (3) of Lemma 3.1. Then by the above construction of $\delta$ the inequality

$$
d_{h}(\pi \circ \gamma(t), \pi \circ \tilde{\gamma}(t))<1
$$

must hold for all $t_{1} \leq t \leq t_{3}$. Consequently $\pi \circ \gamma\left(t_{1}\right)$ and $\pi \circ \gamma\left(t_{2}\right)$ both lie in $A_{n+1}$. The construction of the sequence $\left\{A_{i}\right\}$ yields $\pi \circ \gamma\left(\left[t_{1}, t_{2}\right]\right) \subset$ $A_{n+2}$. The inequality $d_{h}\left(\pi \circ \gamma\left(t_{2}\right), \pi \circ \tilde{\gamma}\left(t_{2}\right)\right)<1$ now yields $\pi \circ \tilde{\gamma}\left(t_{2}\right) \in$ $A_{n+3}$, a contradiction.

This lemma shows that one cannot have a segment of some bicharacteristic curve $\pi \circ \tilde{\gamma}$ of $p^{\prime}$ which leaves $A_{n}$, reaches $A_{n+4}$, and then returns to $A_{n}$. Consequently, a complete bicharacteristic curve $\pi \circ \tilde{\gamma}$ of $p^{\prime}$ which leaves $A_{n}$ and goes to $A_{n+4}$ must eventually reach $A_{n+5}$ after at most returning to $A_{n+1}$.

We now establish the stability of pseudoconvex symbols of real principal type by showing that the set of all symbols in $\operatorname{Smbl}_{k}(X)$ which are both pseudoconvex and of real principal type is an open set in the $C^{1}$-fine topology.

THEOREM 3.3. let $p \in \operatorname{Symbl}_{k}(X)$ for $k \geq 0$ be a pseudoconvex symbol of real principal type. Then there is some $C^{1}$-fine neighborhood $U(p) \subseteq$ $\operatorname{Smbl}_{k}(X)$ such that each $p^{\prime} \in U(p)$ is both pseudoconvex and of real principal type. 
Proof. Let $\delta,\left\{A_{n}\right\}, \varepsilon_{n}$, etc. be as above and set $U(p)=\mathcal{N}(p, 1, \delta, X)$. In order to prove $p^{\prime} \in U(p)$ is of real principal type, let $\tilde{\gamma}:(a, b) \rightarrow T^{*} X$ be an arbitrary complete bicharacteristic strip of $p^{\prime}$. Define $m$ to be the smallest integer such that the image of $\pi \circ \tilde{\gamma}$ intersects $A_{m}$ and assume without loss of generality that $\tilde{\gamma}\left(t_{0}\right) \in S^{*} A_{m}$. The definition of $\varepsilon_{m}$ yields $\pi \circ \tilde{\gamma}\left(t_{1}\right) \in A_{m+5} \backslash A_{m+4}$ for some $t_{1}>t_{0}$. Choose $t_{1}^{\prime} \in\left[t_{0}, t_{1}\right]$ with $\pi \circ \tilde{\gamma}\left(t_{1}^{\prime}\right) \in A_{m+1}$. Lemma 3.2 and the definition of $\varepsilon_{m+1}$ yields some $t_{2}>t_{1}^{\prime}$ with $\pi \circ \tilde{\gamma}\left(t_{2}\right) \in A_{m+6} \backslash A_{m+5}$. Recursively, one may construct a sequence $\left\{t_{n}\right\}$ with $t_{n} \rightarrow b^{-}$and $\pi \circ \tilde{\gamma}\left(t_{n}\right) \in A_{m+4+n} \backslash A_{m+3+n}$. Hence $p^{\prime}$ is of real principal type.

It only remains to show that each $p^{\prime} \in U(p)$ is pseudoconvex. Choose an arbitrary compact subset $K$ of $X$. If $K \subseteq A_{n}$, then Lemma 3.2 implies that any bicharacteristic curve of $p^{\prime}$ with endpoints in $K$ must be in the compact set $A_{n+4}$.

Using the fact that the metric tensor of a Lorentzian manifold is the principal symbol of the d'Alembertian $\square$, we obtain the following corollary which guarantees the $C^{1}$-fine stability of solvability of the Klein-Gordon equation at Lorentzian metrics which are both of real principal type and psuedoconvex.

COROllaRy 3.4. Let $(X, \beta)$ be a Lorentzian manifold such that $\beta$ is both of real principal type and pseudoconvex. There is a $C^{1}$-fine neighborhood $U(\beta)$ of $\beta$ in the space $\operatorname{Lor}(X)$ of all Lorentzian metrics on $X$ such that for each $\beta^{\prime} \in U(\beta)$ the Klein-Gordon equation is solvable on $\left(X, \beta^{\prime}\right)$.

If $(X, \beta)$ is a Lorentzian manifold, the bicharacteristic curves of $\beta$ are the null geodesics of $\beta$ and $\beta$ is of real principal type iff each (inextendible) null geodesic fails to be imprisoned. On the other hand, $\beta$ may be of real principal type and contain a null geodesic which is partially imprisoned in some compact set $I$. This geodesic will have noncompact closure, but leave and return to $K$ an infinite number of times. Of course, if $\beta$ is both of real principal type and pseudoconvex, then partial imprisonment of null geodesics cannot occur. Theorem 3.3 implies that if $\beta$ satisfies both of these conditions, there is a $C^{1}$-fine neighborhood $U(\beta)$ such that each $\beta^{\prime} \in U(\beta)$ fails to have any partial imprisonment of null geodesics.

4. Applications. We begin with some new results on sectional curvatures in pseudoriemannian manifolds $(X, \beta)$ of $\operatorname{dim} \geq 3$. If $\Pi$ is a plane in some fiber of $T^{*} X$, we denote its sectional curvature by $K_{\beta}(\Pi)$. 
In general, $K_{\beta}$ is well-defined only for nondegenerate planes; when $\Pi$ is degenerate, $K_{\beta}$ is "singular" [1, p. 409]. Kulkarni [8] showed that if $K_{\beta}$ is bounded below or above for all nondegenerate planes at $x \in X$, then $X$ has constant curvature at $x$. Several other boundedness conditions which imply constant sectional curvature have also been obtained $[5 ; 6 ; 10]$. Generically, $\left|K_{\beta}(\Pi)\right| \rightarrow \infty$ as $\Pi$ approaches a degenerate plane.

Let $x \in X$ and consider the Grassmann varieties of planes in $T_{x}^{*} X$ and $T_{x} X$, denoted by $G_{2}\left(T_{x}^{*} X\right)$ and $G_{2}\left(T_{x} X\right)$ respectively. We frequently refer to degenerate planes as null planes, and denote them by $n_{2}\left(T_{x}^{*} X\right)$ and $n_{2}\left(T_{x} X\right)$. It is easy to check that $n_{2}$ is a codimension 1 subvariety of $G_{2}$ at each $x$. The varieties $G_{2}$ fit together to form the Grassmann bundles $G_{2}^{*}(X)$ and $G_{2}(X)$ and $K_{\beta}$ is a rational function on $G_{2}^{*}(X)$, or on $G_{2}(X)$ if we change from $\beta$ to the more usual covariant $\bar{\beta}$. Viewed this way, $K_{\beta}$ has poles at almost all null planes; more precisely, in each fiber $K_{\beta}$ has poles at all null planes except for at most a codimension 2 subvariety. Consider $\mathbf{R} P^{1}=\mathbf{R} \cup\{\infty\}$, where one may regard this as the result of identifying $+\infty$ and $-\infty$ in the extended real numbers. If $\Pi_{0}$ is a pole of $K_{\beta}$ we set $K_{\beta}\left(\Pi_{0}\right)=\infty \in \mathbf{R} P^{1}$. If $\Pi_{0}$ is a plane (necessarily null) such that $K_{\beta}(\Pi)$ does not converge in $\mathbf{R} P^{1}$ as $\Pi \rightarrow \Pi_{0}$, we say that $K_{\beta}$ is indeterminate at $\Pi_{0}$ or that $\Pi_{0}$ is an indeterminate plane. At each indeterminate plane the sectional curvature corresponds to the indeterminate type $0 / 0$.

We now temporarily adopt the more traditional viewpoint and work in the tangent bundle $T X$ with the covariant tensor $\bar{\beta}$. Then for $\Pi \in G_{2}(X)$ spanned by $u$ and $v$,

$$
K_{\beta}(\Pi)=\frac{R(u, v, u, v)}{\bar{\beta}(u, u) \bar{\beta}(v, v)-\bar{\beta}(u, v)^{2}} .
$$

where $R$ denotes the Riemann-Christoffel curvature tensor. Now $R(u, v, u, v)$ depends on the particular choice of $u$ and $v$, but its sign depends only on the plane $\Pi$ spanned by $u$ and $v$; in particular, it makes sense to say that $R$ vanishes or is nonvanishing at $\Pi$. An indeterminate plane is always a null plane where $R$ vanishes; however, there are null planes where $R$ vanishes which are not indeterminate.

For completeness and the convenience of the reader, we include the following standard result; the proof is straightforward.

Lemma 4.1. Each pseudoriemannian metric $\beta$ on $X$ has a $C^{0}$-fine neighborhood in $\operatorname{Smbl}_{2}(X)$ which contains only pseudoriemannian structures of the same signature. 
Let $\pi$ denote the natural projection $G_{2}(X) \rightarrow X$.

LEMMA 4.2. Let $K \subseteq G_{2}(X)$ be a compact set on which $R$ is nonvanishing. If $W$ is an open neighborhood of $\pi(K)$ then there exists an open neighborhood $V$ of $K$ and $\varepsilon>0$ such that if $\beta^{\prime} \in \mathscr{N}(\beta, 2, \varepsilon, W)$ then the Riemann-Christoffel curvature tensor $R^{\prime}$ of $\beta^{\prime}$ is nonvanishing on $V$.

Proof. By way of contradiction, suppose not. Then there exist sequences $\varepsilon_{n} \rightarrow 0^{+}$, planes $\Pi_{n} \rightarrow \Pi \in K$, and structures $\beta_{n} \rightarrow \beta$ with $\beta_{n} \in$ $\mathscr{N}\left(\beta, 2, \varepsilon_{n}, W\right)$ such that the curvature tensor $R_{n}$ of $\beta_{n}$ vanishes at $\Pi_{n}$. Let $u$ and $v$ span $\Pi$ and choose sequences $u_{n} \rightarrow u$ and $v_{n} \rightarrow v$ such that $u_{n}$ and $v_{n} \operatorname{span} \Pi_{n}$ for each $n$. Thus $0=R_{n}\left(u_{n}, v_{n}, u_{n}, v_{n}\right) \rightarrow R(u, v, u, v)$ $\neq 0$.

We now show that the nonvanishing of $R$ on a closed $C \subseteq G_{2}(X)$ is a $C^{2}$-fine stable property.

LEMMA 4.3. If $C \subseteq G_{2}(X)$ is closed with $\pi(C)=X$ and $R$ nonvanishing on $C$, then there exists an open neighborhood $V$ of $C$ and $a C^{2}$-fine neighborhood $U(\beta)$ in $\operatorname{Smbl}_{2}(X)$ such that the Riemann-Christoffel tensor $R^{\prime}$ of $\beta^{\prime}$ is nonvanishing on $V$ for each $\beta^{\prime} \in U(\beta)$.

Proof. Let $\left\{L_{i}\right\}$ be a locally finite compact covering of $X$ and set $K_{i}=\pi^{-1}\left(L_{i}\right) \cap C$. Since $G_{2}(X)$ has compact fibers and $C$ is closed, it follows that each $K_{i}$ is compact. Using Lemma 4.2, we obtain a locally finite collection $\left\{V_{i}\right\}$ of open sets with $K_{i} \subset V_{i}$ and positive numbers $\left\{\varepsilon_{i}\right\}$ such that if $\beta^{\prime} \in \mathscr{N}\left(\beta, 2, \varepsilon_{i}, \pi\left(V_{i}\right)\right)$ then its curvature tensor $R^{\prime}$ is nonvanishing on $V_{i}$ for each $i$. Choosing a continuous $\varepsilon: X \rightarrow(0, \infty)$ such that $\varepsilon<\varepsilon_{i}$ on $\pi\left(V_{i}\right)$, letting $U(\beta)=\mathscr{N}(\beta, 2, \varepsilon)$, and setting $V=\cup V_{i}$, the result follows.

In general, negative timelike sectional curvature need not be a $C^{r}$-fine stable condition for $r \geq 0$. Indeed, if $(X, \beta)$ is a model space form of constant curvature (e.g., de Sitter space, Minkowski space, or anti-de Sitter space, in the Lorentzian case), then each $C^{r}$-fine neighborhood $U(\beta)$ contains $\beta^{\prime}$ such that the image under $K_{\beta^{\prime}}$ of the timelike planes is all of $\mathbf{R}$. On the other hand, we can use the Riemann-Christoffel curvature tensor $R$ to describe manifolds for which the condition of negative timelike sectional curvature is $C^{2}$-fine stable. Let $t_{2}\left(T_{x} X\right)$ denote the set of all timelike planes in $T_{x} X$, and define the bundles $t_{2}(X)$ and $n_{2}(X)$ analogous to the definition of $G_{2}(X)$. 
LEMMA 4.4. If $R$ is nonvanishing on $t_{2}(X, \beta) \cup n_{2}(X, \beta)$ then there exists a $C^{2}$-fine neighborhood $U(\beta)$ in $\operatorname{Smbl}_{2}(X)$ such that each $\beta^{\prime} \in U(\beta)$ has a curvature tensor $R^{\prime}$ which is nonvanishing on $t_{2}\left(X, \beta^{\prime}\right) \cup n_{2}\left(X, \beta^{\prime}\right)$.

Proof. Apply Lemma 4.3 with $C=t_{2}(X, \beta) \cup n_{2}(X, \beta)$, noticing that in each fiber $G_{2}\left(T_{x} X\right)$ the set $t_{2}\left(T_{x}(X, \beta)\right) \cup n_{2}\left(T_{x}(X, \beta)\right)$ is closed.

We shall show elsewhere that the nonvanishing of $R$ on $t_{2} \cup n_{2}$ can be used to characterize the $C^{2}$-fine stability of everywhere negative (or positive) timelike sectional curvature.

We now restrict attention to Lorentzian manifolds $(X, \beta)$. In [2] we called $X$ principally causal iff no inextendible causal geodesic was imprisoned. Since the null geodesics of the Lorentzian structure $\beta$ are the bicharacteristic curves of the symbol $\beta$, principally causal implies real principal type. We also called $X$ causally pseudoconvex iff for each compact $K \subseteq X$ there exists a compact $K^{\prime} \subseteq X$ such that each causal geodesic segment of $\beta$ with endpoints in $K$ lies in $K^{\prime}$. Thus causally pseudoconvex implies $\beta$ is a pseudoconvex symbol. These conditions are more restrictive than real principal type and pseudoconvexity, however. Indeed, the cylinder $S^{1} \times \mathbf{R}$ with the Lorentzian structure $\beta$ given by the line element $d s^{2}=d \theta^{2}-d t^{2}$ is not principally causal (because the timelike geodesics $t=$ const. are imprisoned) but $\beta$ is of real principal type (because no inextendible null geodesic is imprisoned). Also, anti-de Sitter space [1, pp. $124 \mathrm{f}$ and 141f] is not causally pseudoconvex but the Lorentzian structure tensor is a pseudoconvex symbol.

The arguments used to prove Theorem 3.3 did not use the fact that the symbol vanishes along a bicharacteristic strip. Thus the same arguments can be applied to principally causal and causally pseudoconvex Lorentzian structures. We state this formally as

Proposition 4.5. If $(X, \beta)$ is a Lorentzian manifold which is principally causal and causally pseudoconvex, then there exists a $C^{1}$-fine neighborhood $U(\beta)$ consisting of principally causal pseudoconvex Lorentzian structures.

The rest of the results in this section now follow from Lemma 4.4 and this proposition, using Theorems 2.4 and 4.6 of [2].

THEOREM 4.6. If $(X, \beta)$ is a principally causal and causally pseudoconvex Lorentzian manifold then there exists a $C^{1}$-fine neighborhood $U(\beta)$ such 
that with respect to any $\beta^{\prime} \in U(\beta)$, the causal convex hull $K K \rrbracket$ of any compact $K \subseteq X$ is compact.

THEOREM 4.7. Let $(X, \beta)$ be a principally causal and causally pseudoconvex Lorentzian manifold of $\operatorname{dim} \geq 3$ with everywhere negative timelike sectional curvature. If the Riemann-Christoffel tensor $R$ is nonvanishing on $n_{2}(X)$ then there exists a $C^{2}$-fine neighborhood $U(\beta)$ such that for each $\beta^{\prime} \in U(\beta)$ and each $x \in X:$

1. the set of points which can be joined to $x$ by a causal curve (including $x)$ is closed;

2. each of the points in (1) can be joined to $x$ by a causal geodesic (degenerate for $x$ itself).

\section{REFERENCES}

[1] J. K. Beem and P. E. Ehrlich, Global Lorentzian Geometry, New York: Marcel Dekker, 1981.

[2] J. K. Beem and P. E. Parker, Klein-Gordon solvability and the geometry of geodesics, Pacific J. Math., 107 (1983), 1-14.

[3] _ The geometry of bicharacteristics and stability of solvability, in Euler Commemorative Volume (ed. G. M. Rassias), to appear.

[4] G. Birkhoff and G. C. Rota, Ordinary Differential Equations, 2nd ed., Waltham: Blaisdell, 1969.

[5] M. Dajczer and K. Nomizu, On the boundedness of Ricci curvature of an indefinite metric, Bol. Soc. Bras. Mat., 11 (1980), 25-30.

[6] S. G. Harris, A triangle comparison theorem for Lorentz manifolds, Indiana Math. J., 31 (1982), 289-308.

[7] M. Hirsch, Differential Topology, G.T.M. 33, New York: Springer-Verlag, 1976.

[8] R. S. Kulkarni, The values of sectional curvature in indefinite metrics, Comm. Math. Helv., 54 (1979), 173-176.

[9] P. W. Michor, Manifolds of Differentiable Mappings, Orpington: Shiva, 1980.

[10] K. Nomizu, Remarks on sectional curvature of an indefinite metric, Proc. Amer. Math. Soc., 89 (19983), 473-476.

[11] F. Trèves, Topological Vector Spaces, Distributions, and Kernels, New York: Academic Press, 1967.

Received May 25, 1983.

UNIVERSITY OF MISSOURI

COLUMBIA, MO 65211

AND

Wichita State University

WICHITA, KS 67208 



\section{PACIFIC JOURNAL OF MATHEMATICS \\ EDITORS}

Donald BABBITT (Managing Editor)

University of California

Los Angeles, CA 90024

J. Dugundu

University of Southern Californa

Los Angeles, CA 90089-1113

R. FINN

Stanford University

Stanford, CA 94305

HermanN FLaSChKa

University of Arizona

Tucson, AZ 85721

\author{
C. C. MOORE \\ University of California \\ Berkeley, CA 94720 \\ ARTHur Ogus \\ University of California \\ Berkeley, CA 94720 \\ Hugo Rossi \\ University of Utah \\ Salt Lake City, UT 84112 \\ H. SAMELSON \\ Stanford University \\ Stanford, CA 94305
}

ASSOCIATE EDITORS
R. ARENS
E. F. BECKENBACH
B. H. NeUmanN
F. WOLF
K. YOSHIDA (1906-1982)

\section{SUPPORTING INSTITUTIONS}

UNIVERSITY OF ARIZONA

UNIVERSITY OF BRITISH COLUMBIA

UNIVERSITY OF OREGON

CALIFORNIA INSTITUTE OF TECHNOLOGY

UNIVERSITY OF CALIFORNIA

MONTANA STATE UNIVERSITY

UNIVERSITY OF SOUTHERN CALIFORNIA

UNIVERSITY OF NEVADA, RENO

STANFORD UNIVERSITY

UNIVERSITY OF HAWAII

NEW MEXICO STATE UNIVERSITY

UNIVERSITY OF TOKYO

UNIVERSITY OF UTAH

WASHINGTON STATE UNIVERSITY

OREGON STATE UNIVERSITY

UNIVERSITY OF WASHINGTON 


\section{Pacific Journal of Mathematics}

\section{Vol. 116, No. $1 \quad$ November, 1985}

K. Adachi, Le problème de Lévi pour les fibrés grassmanniens et les variétés

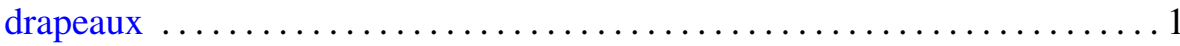

John MacLeod Ball, Remarks on the paper: "Basic calculus of variations" . . . 7 John Kelly Beem and Phillip E. Parker, Whitney stability of solvability . . . 11 Alberto Facchini, Decompositions of algebraically compact modules .......25

S. S. Khare, Finite group action and equivariant bordism $\ldots \ldots \ldots \ldots . \ldots 39$

Horst Leptin, A new kind of eigenfunction expansions on groups $\ldots \ldots \ldots . .45$

Pei-Kee Lin, Unconditional bases and fixed points of nonexpansive

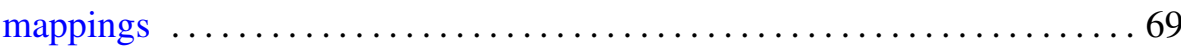

Charles Livingston, Stably irreducible surfaces in $S^{4} \ldots \ldots \ldots \ldots \ldots 77$

Kevin Mor McCrimmon, Nonassociative algebras with scalar involution . . .885

Albert Milani, Singular limits of quasilinear hyperbolic systems in a

bounded domain of $\mathbf{R}^{3}$ with applications to Maxwell's equations

Takemi Mizokami, On $M$-structures and strongly regularly stratifiable

spaces

Jesper M. Møller, On the homology of spaces of sections of complex

projective bundles

Nikolaos S. Papageorgiou, Carathéodory convex integrand operators and

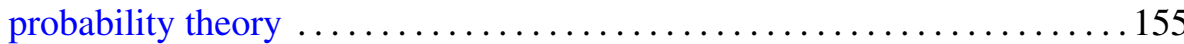

Robert John Piacenza, Transfer in generalized prestack cohomology 185

Lance W. Small and Adrian R. Wadsworth, Integrality of subrings of matrix rings ...

James Michael Wilson, On the atomic decomposition for Hardy spaces 